\title{
CONTICEPTION: A program to study the effects of contingency parameters on judgments of control
}

\author{
PETER J. MCLEOD and ARNOLD B. SPENCE \\ Acadia University, Wolfville, Nova Scotia, Canada
}

\begin{abstract}
Little is known about the heuristics people use in evaluating the degree to which they control events. The DOS-based CONTICEPTION program allows users to independently set the extent to which subjects' actions are necessary and sufficient (the two components of contingency) to cause a change in an animated display. Up to four subject actions (keypresses) can each cause up to four dimensions of the display to change according to independently set contingencies. The task within which subjects judge their control involves interacting with the display in real time. To facilitate modeling of the possible algorithms used by different groups of subjects or under different conditions, the program provides several calculations of contingency magnitude and algebraic rules upon which the literature suggests subjects might base their control judgments. The program also allows sessions (trial sequences) to be saved and provides raw and summarized data output.
\end{abstract}

To know the extent to which we control events in our environment would obviously be adaptive. It is somewhat surprising, therefore, that researchers who have examined subjects' judgments of control or estimates of correlations among discrete events have typically found the accurate assessment of contingent relations to be very difficult, if not impossible. Differences among individuals have been associated with depression (Alloy \& Abramson, 1979) and have also been found between children with learning disabilities and their normally achieving peers (Fuller, 1992; Fuller \& McLeod, in press). Judgments of contingency and control have also been found to be influenced by such extraneous factors as frequency of reward (Alloy \& Abramson, 1979; Jenkins \& Ward, 1965; Wright, 1962), desirability of outcome (Alloy \& Abramson, 1979; Jenkins \& Ward, 1965), skill-related factors (Langer, 1975), foreknowledge of outcome and choice (Wortman, 1975), and social factors (Benassi \& Mahler, 1985). Figure 1 summarizes some of the proposed variables that can influence judgments of control and their relation to the CONTICEPTION program.

Given the complex relations among variables thought to influence control judgments (as illustrated in Figure 1), it is understandable that the heuristics we use in arriving at (often erroneous) judgments of control are still not clearly understood. We believe that the absence of standardized methodologies to conduct judgment of control studies

Development of this program and preparation of this article were supported by a grant from the Natural Sciences and Engineering Research Council of Canada to P.J.M. and by Acadia University. We thank Bill Hayes for help with the final debugging. We gratefully acknowledge comments by Doug Symons on an earlier version of this manuscript and throughout the development of the program. Comments by Sarah Ransdell and other reviewers were also very helpful. Reprint requests should be sent to P. J. McLeod, Department of Psychology, Acadia University, Wolfville, NS, Canada B0P 1 X0 (e-mail: pmcleod@max.acadiau.ca). and the experimental focus on single discrete binary contingencies may also have slowed our progress in this area.

While Mercier, Sweet, and Cheng (1992) have argued that we are best served by simplifying contingency perception experiments (since we are not yet able to model the algorithm[s] used in making the simplest of contingency judgments), "real-world" situations in which such judgments are made are seldom simple. For example, our studies of contingency perception grew out of interests in developmental psychology, where the concepts of contingency and responsiveness (though often not well defined) have played important roles in theories of early cognitive and social development. It has been argued that for infants a key discriminating feature of parents (and social agents in general) is that they behave in a manner that is contingent upon the infant's actions. Watson (1972) has proposed that perceiving these contingent relations may be the most important feature that guides infants to view others as "social objects." The actual schedules of contingent responsiveness that infants are exposed to, however, are not well known, although the concept of contingency has frequently been used in the examination of maternal responsiveness. (See Symons \& Moran, 1994, for explicit measures of contingency magnitude during mother-infant face-to-face interactions.)

Part of the problem in describing these social contingencies is that there are many possible behaviors that each interactant can use in a responsive way. Social exchanges do not involve a one-to-one correspondence between participants' actions. In addition to the multimodal nature of the social contingencies which are assumed to influence infants, parents provide infants with very high levels of spontaneous (noncontingent) stimulation. We also do not know how sensitive infants are in discriminating among different contingent schedules, nor which contingencies, if any, tend to be preferred at different ages. 


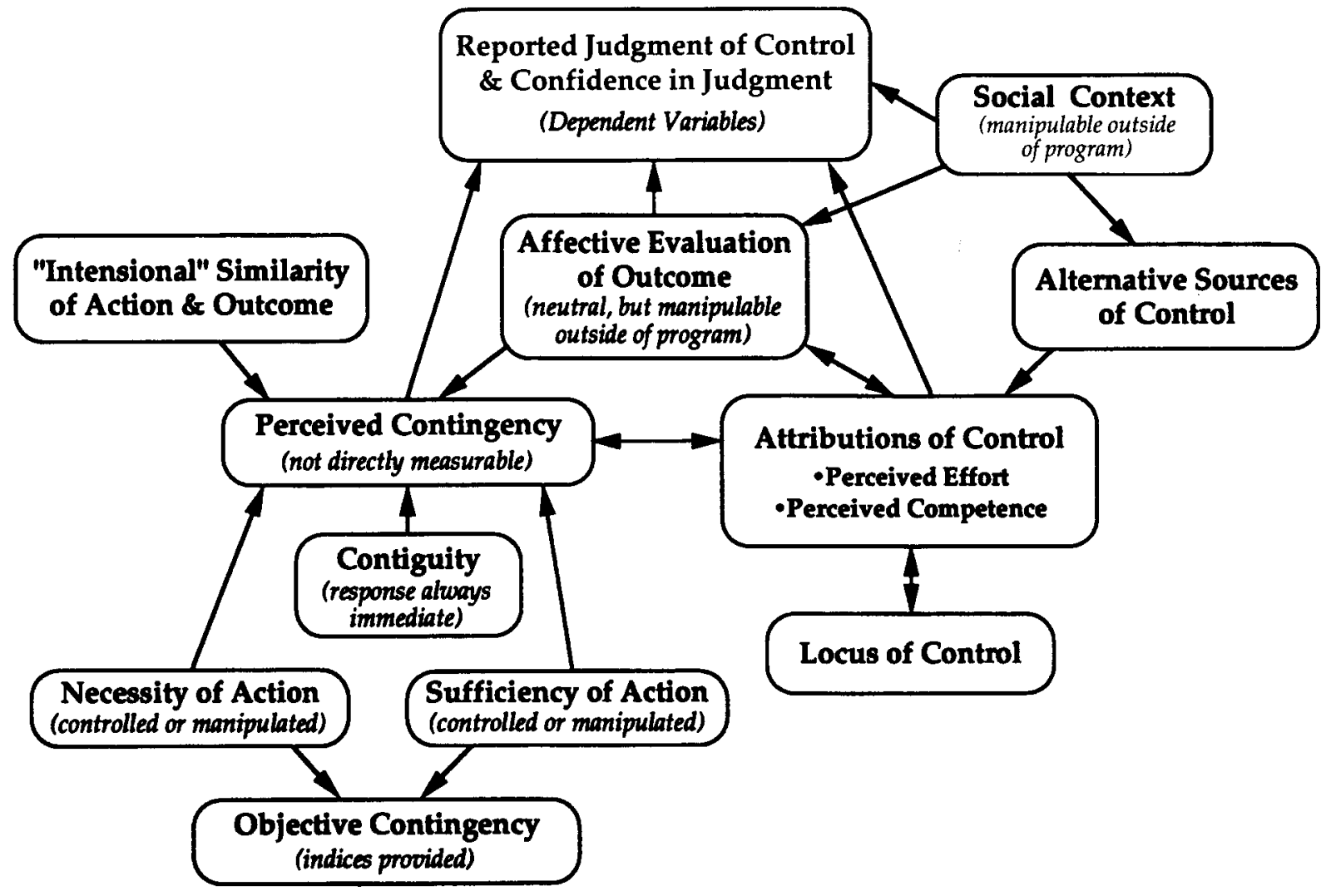

Figure 1. Schematic illustration of some of the variables shown or hypothesized to influence human judgments of control and suggested directions of those influences. Note that this figure is not intended to be a model of the process of making such judgments and that the spatial arrangement of the items was determined solely on the basis of visual simplicity. Some of the variables incorporated into experiments with the CONTICEPTION program and the nature of their involvement are indicated in parentheses.

Watson (1979) has also argued that it is important to distinguish among the different structural forms that contingent relations can have (the relative contributions of necessity and sufficiency to a behavior-response contingency), because they probably have somewhat different effects on how otherwise equivalent contingencies are perceived. To the best of our knowledge, however, there are no readily and widely available methods to manipulate these parameters in contingency perception experiments. Rather, a review of the literature suggests that researchers develop and use idiosyncratic tasks to collect data.

We hope that the flexibility provided by the CONTICEPTION program in providing contingent schedules between subjects' actions and outcomes will make it attractive to a wide range of users. The complex contingent relations that this program can provide will also facilitate the examination of higher order relations among variables that are likely involved in many real-world situations (such as social contingencies). The approach of starting with very simple contingent tasks, advocated by Mercier et al. (1992), has certainly been fruitful, and it may answer questions about the relative contribution of necessity and sufficiency to the perception of binary contingencies. Complementary research programs employing more multivariate designs are also needed, how- ever, to model social contingencies. Since the relations among the variables in Figure 1 are unlikely to be additive, multivariate approaches may be necessary for understanding even the simplest contingency judgments. Although complex contingencies such as those which CONTICEPTION can provide are more reflective of some real-world situations and in this sense are more ecologically valid, the task itself is quite artificial. Still, this program can be used in both simple and complicated experimental tasks.

CONTICEPTION is a compiled program, written in Turbo Pascal (Version 5.5), which provides the experimenter with a series of windows within which the parameters of real-time contingency judgment experiments can be set and saved. This program allows the experimenter considerable flexibility in determining the contingency schedules among subjects' keypressing behavior and the changes that occur in the movement and appearance of a disk displayed on the computer monitor. The key capabilities of this program are described below.

The number of keys that the program will recognize as acceptable input can be set from one (as has been used in most previous studies) to four. Previous work by Wasserman (1990) has shown that under some conditions subjects are able to give quite accurate concurrent re- 
ports for two separate responses. The ability of the present program to use up to four subject actions will allow further explorations of the limits of human processing of this type of information and provide an experimental analogue for researchers interested in perceptions of the contingent environments found in social exchanges (where, for example, smiles, vocalizations, head nodding, and eyebrow movements may all be appropriate responses).

The number (up to four) and nature of the dimension(s) of the animated display that is(are) contingently responsive to each input can be selected. Each of these dimensions has two states (determined by the experimenter for three of these dimensions) which can alternate either responsively or spontaneously. The four dimensions are: (1) color of the moving disk ( 2 of 16 colors can be selected for the color states by the experimenter) ${ }^{1}$; (2) $d i$ rection in which the disk travels (clockwise or counterclockwise); (3) orbit followed by the disk (the size and shape of both trajectories can be set by the experimenter); and (4) the speed with which the disk moves (the two speeds are infinitely variable up to the maximum determined by the processing speed of the platform on which the program is run). This flexibility allows the user to examine a person's judgments about situations in which his/her actions may or may not always lead to the same response. Again this allows more valid modeling of social contingencies (wherein a smile, for example, might be responded to in a number of possible ways). In contrast to the fourth rule of Hume's (1739/ 1978) mechanistic account of human causal perception, which requires the same cause to always produce the same effect, our studies with this variable have suggested that, under certain conditions at least, control judgments are not influenced by the number of possible responsive dimensions (McLeod, 1993). The second component of Hume's rule, that the same effect never arises but from the same cause, could also be examined with CONTICEPTION by having more than one acceptable input causing the same dimension of the animation to change.

Responsiveness (sufficiency, or the probability with which an action [a keypress] will cause an outcome [a change in a dimension]) can independently be set from $0 \%$ to $100 \%\left(0.0 \leq p_{\text {response }} \leq 1.0\right)$ for each active key. ${ }^{2}$

When more than one dimension is set to respond to a given key, the relative probabilities by which each chosen dimension will respond can be set by the experimenter. Each dimension can also change pseudorandomly (independently of subjects' keypressing) at rates set by the user. This allows control over the second component of contingency, necessity (the extent to which actions must precede outcomes).

Trial duration can either be set by the experimenter or determined by each subject, and a screen of instructions (written by the experimenter) can be shown to subjects prior to a session. Subjects can also be given on-line visual feedback during trials about the number of times they have pressed each relevant key. This feature can be used to assist subjects in acting equally often among trials so that differences in control judgments are not con- founded by differences in the amount of information obtained prior to subjects' making their judgments.

Session lists of trials (i.e., sequences of set-up files specifying the contingency conditions of each successive trial) can be saved, thereby simplifying counterbalancing for order effects in mixed- and within-subjects designs. This also allows experimentation on the effects of previous contingent experiences on judgments of control. For example, by incrementing the contingent levels from trial to trial, the magnitude and persistence of any carry-over effects of having experienced low levels of control can be determined. Similarly, carryover effects of decreasing contingencies over trials can be examined. Data from each trial for each subject are saved in separate files, which can later be summarized on screen and printed.

In addition to the flexibility that these features allow for setting up experiments, it is important to note that subjects are asked to make control judgments about events that occur in real-time. While this makes precise calculations of the actual degree of contingency (e.g., a phi $\left[r_{\phi}\right]$ coefficient) problematic, relative judgments under different parameter settings can be compared under more ecologically valid conditions than is the case with tasks that divide time into sequences of discrete units of arbitrary duration (much like chess artificially represents a battle as an alternating sequence of moves). The task for subjects using CONTICEPTION is to interact in real time with the program in order to determine their control over changes in an animation. The dependent variable typically used would therefore be subjects' verbal judgments of the extent to which they are in control of the animation during the trial (see Figure 1). Previous work with this program has also found subjects' confidence in their judgments of control to reveal differences between groups that do not appear in their judgments of control (e.g., with age-Cain, 1992; Cain \& McLeod, 1992; and between students with learning disabilities and those without-Fuller, 1992; Fuller \& McLeod, in press). Confidence judgments can also be influenced by variables (e.g., gender) that do not vary directly with control judgments (McLeod, 1994). For many researchers, perceived contingency (rather than reported judgments of control) will be the dependent variable of interest, although there is no way to measure this directly.

For those interested in modeling subjects' control judgments or obtaining indices of judgment accuracy (cf. Allan, 1980; Allan \& Jenkins, 1983; Shanks, 1985; Wasserman, 1990), CONTICEPTION provides several relevant calculations based on the cell frequencies of a $2 \times 2$ contingency table. In addition to counting rules such as the number of successes [cell $a$ (action $\rightarrow$ outcome)], or total number of outcomes [cells $a+c$ (no action $\rightarrow$ outcome)], differences in the frequency of observations consistent and inconsistent with contingency are also provided $[\Delta f=(a-c)$ and $(b-d)$, where $b$ is the frequency for the cell "action $\rightarrow$ no outcome" and $d$ for the cell "no action $\rightarrow$ no outcome"], as are the percent success $[a /(a+b)]$ and the more complicated $\Delta d\{\Delta d=[(a+d)-$ 
$(b+c)]\}$, normative $\Delta p$ rule $\{\Delta p=[a /(a+b)-c /(c+d)]$, and phi coefficient $\left\{r_{\phi}=|a d-b c| / \sqrt{ }[(a+b)(c+d)(a+c)\right.$ $(b+d)]\}$.

Of crucial importance to these more complicated calculations is determining the frequency for cell $d$. There are no a priori bases for determining the relevant time unit for determining chance probabilities with real-time events (Wasserman, 1990; Watson, 1979). Although a 1sec time unit has been used in the past (e.g., Wasserman, 1990), interpretations of the results from such analyses must be made tentatively and without masking the subjectiveness of the time unit used. To directly address this issue, the CONTICEPTION program allows the user to specify and alter (post hoc) the computational time unit and see the resulting effects on the contingency calculations.

To assist in selecting a psychologically relevant unit, the program also provides two subject-defined intervals. The first is the minimum interval between successive actions by the subject. The use of this duration assumes that subjects would not act twice within a single subjectively determined sampling period but might choose to act in two successive time samples. The second calculated interval is based on an assumption that normatively minded subjects would likely look for consequences of their actions as frequently as they look for baseline activity. From this assumption, the time between the start of the trial and the subject's last keypress is divided by $2(a+b)$ to obtain another suggested sampling time unit for individual subjects.

The number of variables that can be controlled while using CONTICEPTION allows the experimenter to create extremely complex contingency problems for subjects to judge. For example, it is possible to have the program create an animated display that is probabilistically responsive to four different subject actions, some (or all) of which may have up to four dimensions responding (at different probabilities), and each dimension (whether responsive or not) may also randomly change at different rates. It is our belief that this complexity and flexibility will be extremely useful for exploring the limits of human processing of probabilistic causal information.

Availability. The CONTICEPTION program is available on either a 5.25 - or a 3.5 -in. flexible disk (containing an executable, compiled version of the program; sample set-up and text [instruction] files; and an instruction manual) from the first author for $\$ 25.00$. This program will run on any DOS-based platform; its performance will vary with the speed and graphic capabilities of the computer being used.

\section{REFERENCES}

ALLAN, L. G. (1980). A note on measurement of contingency between two binary variables in judgment tasks. Bulletin of the Psychonomic Society, 15, 147-149.

Allan, L. G., \& Jenkins, H. M. (1983). The effect of representations of binary variables on judgment of influence. Learning \& Motivation, 14, 381-405.

Alloy, L. B., \& ABramson, L. Y. (1979). Judgment of contingency in depressed and nondepressed students: Sadder but wiser? Journal of Experimental Psychology: General, 108, 441-485.

Benassi, V. A., \& MaHLER, H. I. M. (1985). Contingency judgments by depressed college students: Sadder but not always wiser. Journal of Personality \& Social Psychology, 49, 1323-1329.

CAIN, M. (1992). The development of contingency perception and perceived control. Unpublished honors thesis, Acadia University, Wolfville, NS.

CAIN, M., \& MCLEOD, P. J. (1992). Social context and the development of perceived control. Canadian Psychology, 33, 375.

FULLER, M. (1992). Contingency perception and beliefs about control in students with learning disabilities. Unpublished master's thesis, Acadia University, Wolfville, NS.

FULLER, M., \& MCLEOD, P. J. (in press). Judgements of control over a contingently responsive animation by students with and without learning disabilities. Canadian Journal of Behavioural Science.

Hume, D. (1978). A treatise of human nature (2nd ed.). Oxford: Oxford University Press, Clarendon Press. (Originally published 1739)

JENKINS, H. M., \& WARD, W. C. (1965). Judgment of contingency between responses and outcomes. Psychological Monographs, 79, (1, Whole No. 594).

LANGER, E. J. (1975). The illusion of control. Journal of Personality \& Social Psychology, 32, 311-328.

MCLEOD, P. J. (1993). Judgments of control over a contingently responsive animation. Paper presented at the meeting of the Canadian Society for Brain Behaviour and Cognitive Sciences, Toronto.

MCLEOD, P. J. (1994). Age differences in judgments of control. Poster presented at the meeting of the International Society for the Study of Behavioral Development, Amsterdam,

Mercier, P., Sweet, L., \& Cheng, Y. (1992). The computation and representation of on-line contingency estimation. Paper presented at the meeting of the Canadian Society for Brain Behaviour and Cognitive Sciences, Quebec

SHANKS, D. R. (1985). Continuous monitoring of human contingency judgment across trials. Memory \& Cognition, 13, 158-167.

SYMONS, D. K., \& MORAN, G. (1994). Responsiveness and dependency are different aspects of social contingencies: An example from mother-infant smiles. Infant Behavior \& Development, 17, 209-214.

WASSERMAN, E. A. (1990). Detecting response-outcome relations: Toward an understanding of the causal texture of the environment. Psychology of Learning \& Motivation, 26, 27-82.

Watson, J. S. (1972). Smiling, cooing, and "The Game." MerrillPalmer Quarterly, 18, 323-339.

WATSON, J. S. (1979). Perception of contingency as a determinant of social responsiveness. In E. B. Thoman (Ed.) The origins of social responsiveness (pp. 33-64). Hillsdale, NJ: Erlbaum.

Wortman, C. B. (1975). Some determinants of perceived control. Journal of Personality \& Social Psychology, 31, 282-294.

Wright, J. C. (1962). Consistency and complexity of response sequences as a function of schedules of noncontingent reward. Journal of Experimental Psychology, 63, 601-609.

\section{NOTES}

1. Not available on monochrome systems.

2. Note that due to the randomization procedures used in the CoNTICEPTION program, actual responsiveness may vary slightly from the specified value. For this reason, the program also calculates the actual responsiveness as part of the summarized data screen.

(Manuscript received August 7, 1992; revision accepted for publication July 27,1994 .) 